\title{
Preclinical and subclinical events in motor neuron disease
}

\author{
MICHAEL SWASH, DAVID INGRAM \\ From the Department of Neurology, The London Hospital, London, UK
}

SUMMARY It is suggested that in motor neuron disease there is a long preclinical period of relative tolerance and compensation before presentation with apparently focal features. During this phase the disease becomes disseminated through the motor system. The mode of acquisition of the disease, its relation to a possible genetic factor, and the processes leading to tolerance, to latency or progression, to the relative involvement of upper and lower motor neurons, to involvement of spinocerebellar pathways, and to asymmetry are fundamental problems in understanding the disease.

Motor neuron disease usually presents with weakness, atrophy or muscle cramp. Less commonly fasciculation, gait disorder, dysarthria or respiratory difficulty may be the first disability. ${ }^{1-6}$ These presenting features conceal fundamental problems in understanding the disease, particularly the initial events, the duration of the subclinical phase of the disease process prior to presentation, and factors leading to the variable and often focal pattern of initial involvement and progression of the disease. Clinical observations in patients with motor neuron disease, taken together with other evidence, suggests that there may be a long pre-clinical phase of months or even several years duration during which the disease is not symptomatic, and that a latent phase may develop even when the illness is established. In this paper these concepts are discussed in relation to the case histories of three patients with motor neuron disease.

\section{Patients}

Three patients, selected from a consecutive group of 31 patients entered into a prospective study of the nature, clinical pattern and progression of the disease, will be described.

Address for reprint requests: Dr M Swash, The London Hospital, Whitechapel, London El IBB, UK.

Received 21 August 1987. Accepted 22 September 1987

\section{Case 1}

This $\mathbf{4 2}$ year old army officer presented with weakness of the left foot. For 2 years he had noted cramp in calf, shoulders, and abdominal muscles on exercise with a tendency to limp slightly when fatigued. For 6 months he had tended to catch the left foot when walking on rough ground and had noticed slight difficulty climbing stairs and rising from a low chair. In addition he had noted muscular twitching in abdominal, neck, left shoulder and facial muscles.

Examination revealed mild asymmetrical weakness particularly involving right deltoid, both triceps and thenar muscles, trunk and distal leg muscles. The left leg was more severely affected than the right, with slight distal atrophy. The tendon reflexes were all increased especially in the left leg. There was spasticity in both legs and both plantar responses were extensor. Fasciculations were observed in all four limbs and in the tongue. Sensory examination was normal. EMG showed widespread neurogenic changes in all four limbs with normal motor and sensory conduction. The CSF was normal. The creatine kinase level was $818 \mathrm{IU} / 1$ (normal less than $160 \mathrm{IU} / \mathrm{l}$ ). A muscle biopsy showed grouping of both fibre types with clusters of atrophic denervated fibres.

When the diagnosis was discussed with the patient it became apparent that there had been a long preliminary phase to the illness before the development of definite weakness, atrophy, cramp or fasciculations. In the Army he had been required to pass an annual physical test consisting of a timed one mile $(1.6 \mathrm{~km})$ run carrying a $60 \mathrm{lb}(28 \mathrm{~kg})$ backpack. He had first failed this test 6 years before, at the age of 35 years, but with extra training had achieved the required standard. He then found that in order to pass this test each year he required several training sessions. He had 
sought medical advice only when he was unable: to pass this test, and when the left foot became weak. In the 18 months subsequent to the diagnosis of motor neuron disease he rapidly weakened, to the point that he required wheelchair assistance outdoors.

Case 2

This 34 year old policeman presented with progressive weakness of the right leg. At the age of 29 years, during a period of 6 weeks, he had developed a right wrist drop. At that time there was weakness of wrist and finger extension with fasciculation. There was no weakness of wrist or finger flexion and the triceps and shoulder muscles were normal. No sensory, or other abnormality was detected. EMG showed neurogenic changes in the forearm extensor muscles but no change in motor latency in the radial nerve. A tendon transfer procedure produced functional improvement. Two years later, aged 31 years, he noted fasciculation in the right quadriceps muscle; within a few weeks weakness of dorsiflexion of the right foot developed, followed by weakness of right knee extension. After a few months this improved and he remained well and able to take part in all activities, including sport. However, a further 2 years later he noted frequent cramp in the right leg, right hand and abdominal muscles with slowly progressive weakness of the right leg and hand and slurring of speech. These symptoms worsened after exertion.

Examination revealed weakness and atrophy of the right wrist extensors, triceps and small hand muscles, with some involvement of small hand muscles on the left, and of the right deltoid muscles. In the legs there was weakness and mild atrophy in the extensors at the ankle, gastrocnemius and quadriceps, more evident on the right than on the left. He was unable to hop on the right foot. Widespread fasciculation was noted in all four limbs and in the tongue. Tone was increased in the right leg and both plantar responses were extensor. There was no sensory abnormality: EMG showed widespread neurogenic change with fibrillation and fasciculation potentials, but, with normal motor and sensory nerve conduction. The creatine kinase level was 377 IU/1 (normal less than $160 \mathrm{IU} / \mathrm{l}$ ) and a quadriceps muscle biopsy revealed neurogenic changes. In the subsequent year there was rapid progression in weakness and atrophy and he developed an increasingly severe bulbar palsy, with marked functional disability. These symptoms have prevented him working.

\section{Case 3}

This 77 year old woman had noted progressive weakness of the ankles affecting the left more than the right, beginning about 15 years previously. Examination revealed asymptomatic proximal weakness in the upper limbs, with infrequent fasciculations in all four limbs and in the trunk musculature. No bulbar abnormality was noted. The tendon reflexes were reduced in the legs, but both plantar responses were extensor. Muscular tone was normal. There was no sensory impairment. EMG revealed widespread neurogenic changes with fibrillations and fasciculation potentials, but with normal motor and sensory nerve conduction velocity. The terminal motor latencies in the common peroneal, median and ulnar nerves were slightly increased. Myelography revealed mild cervico-lumbar spondylosis without cord compression, and CT of the brain and of the cervical region was normal. The CSF was normal.

\section{Discussion}

In motor neuron disease degeneration of the upper and lower motor neurons causes a combination of upper and lower motor neuron signs. These clinical features vary in their distribution and severity. Striking asymmetry and selective involvement. of individual groups of muscles, especially of hand and forearm, and of foot and lower leg, are characteristic early features of the disease. ${ }^{17}$ Furthermore, the rate of progression of the disease shows considerable variability, as shown by estimates of survival ranging from 3 months to more than 7 years from diagnosis. ${ }^{8}$ Mulder and Howard ${ }^{9}$ suggested that these clinical variations in the disease indicated the interaction of factors responsible for relative resistance and susceptibility. They described a patient in whom the disease had remitted, other diagnoses having been excluded by clinical investigation. This appears to be the only report of remission and recovery from motor neuron disease. ${ }^{9}$ The three patients described here illustrate several important aspects of the disease that have been neglected in recent reviews. ${ }^{5} 610$

First, the initial event in the disease is unknown. Presentation with weakness, wasting and fasciculation, even if focal, implies extensive loss of motor neurons in the pool of anterior horn cells innervating wasted muscles. Both weakness and wasting are features of lower motor neuron involvement, but the extent of this involvement may be masked by the? capacity for reinnervation of the remaining motor neurons. Wohlfart ${ }^{11}$ showed that weakness and atrophy were detectable only when $30 \%$ or more of the anterior horn cells innervating a group of muscles were-lost. Recent postmortem studies of the number of motor neurons remaining in lumbar and cervical segments $^{13}$ in patients dying with motor neuron disease show that about a third of cells remain.

Secondly, loss of cells in the cord is markedly focal and asymmetrical even in the terminal stages of the disease. $^{13}$. Thus there is selective involvement of motor neurons in the anterior horns of the cord both early and late in the disease, an observation that must reflect local variations in susceptibility and resistance to the disease. ${ }^{913-15}$ The clinical features suggest that this selectivity is variable from case to case, implying that susceptibility and resistance to the disease depend on the interaction of several different factors. The corticospinal pathways in the cord are similarly asymmetrically affected. ${ }^{16}$ Some motor neurons show selective resistance to the disease. For example, the motor cells of Onuf's sacral nucleus that innervate the anal and urinary striated sphincter muscles are always 
spared $^{17} 18$ and the ocular motor nuclei are also relatively resistant. ${ }^{510}$

Thirdly, the kinetics of cell loss in the disease are unknown. Patterns of clinical deterioration vary from rapid to slow, and from inexorable to episodic and rapid, as in Cases 1 and 2. The clinical features of the disease are the result of the inter-relation of denervation from loss of functional motor neurons, reinnervation by collateral sprouting from the motor unit territories of neighbouring motor neurons, and supranuclear effects from damage to the upper motor neuron pathway. Hansen and Ballantyne ${ }^{19}$ suggested that collateral sprouting remained effective only as long as the territories of motor units remained in contact and calculated that reinnervation was maximal when $50 \%$ of anterior horn cells remained. They suggested that reinnervation ceased when less than $5-10 \%$ of cells remained. Postmortem studies suggest that rather more than this number of cells may remain in most cases. ${ }^{12}{ }^{13}$ Nonetheless, the clinical evidence suggests a wide range of susceptibility to motor neuron loss, not only from case to case but at different levels of the cord. ${ }^{13}$ Furthermore, loss of cells is not related to individual muscles but tends to involve part of a limb, usually affecting the territories of several cord segments. ${ }^{13}$ Since muscle spindles show loss of both gamma and beta motor innervation in histological studies ${ }^{25}$ it is apparent that both the small gamma and large alpha motor neurons are involved in the disease.

Fourthly, EMG data on the distribution of reinnervation suggests that the disease is widespread at the time of diagnosis, implying that it has been active and disseminated through the motor system for some time prior to presentation with weakness and atrophy. ${ }^{714} 15$ The characteristic widespread distribution of fasciculation often noted at the time of presentation $^{5610}$ as in Cases 1 and 2, also suggests that there is a long phase of pre-clinical involvement of motor neurons prior to the development of weakness. This early phase of relative resistance is exemplified in Case 1, in whom fatiguability, leading to inability to complete a physical task that he had formerly found easy, was a feature for 6 years prior to the development of weakness and atrophy. During these 6 years, physical training was capable of restoring function, and the only other sign of abnormality in the motor system was a tendency to develop cramp on exertion. Similar observations were made by Fleet and Watson ${ }^{21}$ in a man who had cramps and fasciculation for 4 years prior to the development of typical motor neuron disease.

Fifthly, a latent or arrested stage may develop. ${ }^{5} 9$ Case 2 presented with a rapid onset of weakness in the radial extensors. This stabilised for 2 years, without further disability before weakness developed in a leg.
This improved substantially and he was well and capable of hard physical work as a policeman for a further 2 years before the disease entered a rapidly progressive phase, with more widespread involvement leading to severe disability in a few months. The presence of widespread cramp and fasciculation suggest that there was widespread involvement during most of this time, but that it was well compensated. It is difficult to decide whether the later rapid deterioration was due to overloading of compensatory mechanisms in the cord or muscle, or whether it resulted from breakdown in patient resistance, with consequent rapid loss of functioning motor neurons. The abrupt loss of previously well-compensated, large, reinnervated motor units may produce disproportionate weakness, and the clinical picture of rapid, preterminal deterioration. Case 3 showed high overall resistance to the disease with slow progression during many years.

Sixthly, pathological studies have shown that involvement of spinocerebellar pathways is an almost invariable, although clinically silent, feature of the disease. $^{22}$ The cells of Clarke's column, forming the nucleus of the dorsal spinocerebellar tract, are regularly involved ${ }^{23}$ and Marchi studies show asymmetrical involvement of both ventral and dorsal spinocerebellar pathways in the cord. ${ }^{24}{ }^{25}$ No relation between involvement of lower and upper motor neurons is yet apparent. ${ }^{5} 10$

\section{Conclusions}

These features are important in understanding motor neuron disease. They are consistent with a disease process that presents with relatively focal weakness, atrophy and spasticity after a long preclinical period during which the disease has become disseminated in the motor system. During the course of the disease periods of relative susceptibility or resistance can sometimes be recognised. Currently, there is no clue as to the mode of acquisition or onset of the disease. ${ }^{26}$

We thank the Amyotrophic Lateral Sclerosis Association (USA), the Motor Neurone Disease Association (UK) and The London Hospital Special Trustees for financial support.

\section{References}

1 Jokelainen M. Amyotrophic lateral sclerosis in Finland, Part 2 clinical characteristics. Acta Neurol Scand 1977;56:194-204.

2 Sivak ED, Streib EW. Management of hypoventilation in motor neuron disease presenting with respiratory insufficiency. Ann Neurol 1980;7:188-91.

3 Mortara P, Chio A, Rossa MG, Leone M, Schiffer D. Motor neuron disease in the province of Turin, Italy 1966-1980. J Neurol Sci 1984;66:165-73. 
4 Caroscio JT, Calhoun WF, Yahr MD. Prognostic factors in motor neuron disease: a prospective study of longevity. In: Rose FC (ed) Research Progress in Motor Neurone Disease. London: Pitman 1984:34-43.

5 Mulder DW. Clinical limits of amyotrophic lateral sclerosis. In: Rowland LP (ed). Human Motor Neuron Diseases. New York: Raven Press 1982:15-22.

6 Gubbay SS, Kahana E, Zilber N, Cooper G, Pintor S, Liebowitz Y. Amyotrophic lateral sclerosis: a study of its presentation and prognosis. J Neurol 1985;232:295-300.

7 Schwartz MS, Swash M. Pattern of involvement in the cervical segments in the early stage of motor neuron disease-a single fibre EMG study. Acta Neurol Scand 1982;65:424-31.

8 Jergens SM, Kurland LT, Okazaki H, Mulder DW. ALS in Rochester, Minnesota 1925-1977. Neurology 1980;30:463-70.

9 Mulder DW, Howard EM. Patient resistance and prognosis in amyotrophic lateral sclerosis. Mayo Clin Proc 1976;51:537-41.

10 Tandan R, Bradley WG. Amyotrophic lateral sclerosis: part 1, clinical features, pathology and ethical issues in management. Ann Neurol 1985;18:271-80.

11 Wohlfart G. Collateral reinnervation in partially denervated muscle. Neurology 1958;8:175-80.

12 Tsukagoshi $H$, Yanagisawa $K$, Oguchi, Nagashina $K$, Murakami T. Morphometric quantification of the cervical limb motor cells in controls and in amyotrophic lateral sclerosis. $J$ Neurol Sci 1979;41:287-97.

13 Swash M, Leader M, Brown A, Swettenhan K. Focal loss of anterior horn cells in the cervical cord in motor neuron disease. Brain 1986;109:939-52.

14 Swash M. Vulnerability of lower brachial myotomes in motor neuron disease: a clinical and single fibre EMG study. $J$ Neurol Sci 1980;47:59-68.

15 Swash M, Schwartz MS. A longitudinal study of changes in motor units in motor neuron disease. J Neurol Sci 1982;56:
185-97.

16 Ingram DA, Swash M. Central motor conduction is abnormal in motor neuron disease. $J$ Neurol Neurosurg Psychiatry 1987;50:159-66.

17 Mannen T, Iwata M, Toyokura Y, et al. Preservation of a certain motor neuron group of the sacral cord in amyotrophic lateral sclerosis: its clinical significance. J Neurol Neurosurg Psychiatry 1977;40:464-9.

18 Schroder HD, Reske-Nielsen E. Preservation of the nucleus $x$ pelvic floor motosystem in amyotrophic lateral sclerosis. Clin Neuropathol 1984;3:210-6.

19 Hansen S, Ballantyne JP. A quantitative electrophysiological study of motor neuron disease. J Neurol Neurosurg Psychiatry 1978;41:773-83.

20 Fleet WS, Watson RT. From benign fasciculations and cramps to motor neuron disease. Neurology 1986;36:997-8.

21 Brownell B, Oppenheimer DR, Hughes JT. The central nervous system in motor neuron disease. J Neurol Neurosurg Psychiatry 1970;33:338-57.

22 Averback P, Crocker P. Regular involvement of Clarke's nucleus in sporadic amyotrophic lateral sclerosis. Arch Neurol 1982;38:155-6.

23 Smith MC. Nerve fibre degeneration in the brain in amyotrophic lateral sclerosis. J Neurol Neurosurg Psychiatry 1960;23:269-82.

24 Swash M, Scholtz CL, Vowles G, Ingram DA. Asymmetrical involvement of crossed and uncrossed corticospinal tracts and spinocerebellar tracts in motor neuron disease. $J$ Neurol Neurosurg Psychiatry (in press.)

25 Swash M, Fox KP. The pathology of the muscle spindle: effect of denervation. J Neurol Sci 1974;22:1-24.

26 Calne DB, Eisen A, McGeer E, Spencer P. Alzheimer's disease, Parkinson's disease and motor neuron disease: abiotrophic $\mathbb{D}$ interaction between ageing and environment? Lancet 1986; ii: $1067-70$. 